\title{
Biomechanical evaluation of intra-articular and extra-articular procedures in anterior cruciate ligament reconstruction: A finite element analysis
}

\author{
N.A. Ramaniraka ${ }^{\text {a }}$, P. Saunier ${ }^{\text {a }}$, O. Siegrist ${ }^{\text {b }}$, D.P. Pioletti ${ }^{\mathrm{a}, *}$

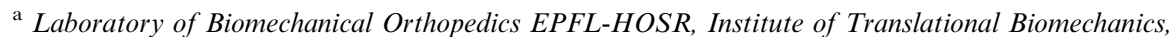 \\ Ecole Polytechnique Fédérale de Lausanne, Station 15, 1015 Lausanne, Switzerland \\ ${ }^{\mathrm{b}}$ Hôpital Orthopédique de la Suisse Romande, Avenue Pierre-Decker, 4, 1005 Lausanne, Switzerland
}

Received 3 April 2006; accepted 17 October 2006

\begin{abstract}
Background. Intra-articular techniques (single and double bundles) are the most widely used procedures for the anterior cruciate ligament reconstruction. Lemaire introduced in 1967 the extra-articular techniques, and combined intra-articular and extra-articular reconstruction, to better restore the stability of the knee. However, the effectiveness of these procedures (intra-articular, extra-articular combined or not with intra-articular) seems to be controversial.

Methods. In the present study, we developed numerical models of a knee joint to evaluate the effects of these different procedures on the kinematics and biomechanics of the knee during an internal rotation test. Six cases were simulated: intact anterior cruciate ligament, intra-articular reconstructed anterior cruciate ligament (single and double bundles), extra-articular reconstructed anterior cruciate ligament alone, and combination of extra- and intra-articular reconstructions. The loading condition was an internal tibial torque of $2 \mathrm{~N}$ m at $0^{\circ}, 15^{\circ}, 30^{\circ}$ and $45^{\circ}$ of knee flexion. Internal rotation of the tibia and forces within the grafts and the ligaments were calculated.

Findings. This study showed that both single and double bundles intra-articular reconstructions restore similar internal rotation control and biomechanics of the soft structures as the intact anterior cruciate ligament situation. On the other hand, our results indicate that extra-articular reconstruction reduces appreciably the internal rotation and modifies the charges distribution in the soft structures when compared to the intact anterior cruciate ligament.

Interpretation. The extra-articular procedure alters the kinematics of the knee, which might overconstraint the ligaments and the femorotibial joints, leading to the failure of the anterior cruciate ligament reconstruction.
\end{abstract}

(C) 2006 Elsevier Ltd. All rights reserved.

Keywords: Extra-articular; Anterior cruciate ligament reconstruction

\section{Introduction}

The primary functions of the anterior cruciate ligament (ACL) are to prevent the anterior displacement of the tibia with respect to the femur, and to constrain the internal rotation of the tibia. The ACL consists of two bulks: the anteromedial bulk essentially controls the anterior tibial

\footnotetext{
${ }^{*}$ Corresponding author.

E-mail address: dominique.pioletti@epfl.ch (D.P. Pioletti).
}

translation, and the posterolateral bulk is thought to control the rotatory stability of the knee (Christel et al., 2005).

Several techniques are used for anterior cruciate ligament reconstruction, the intra-articular procedures being the mostly used. The single bundle (1B) intra-articular procedure intends to replace the anteromedial bulk, while the double bundles (2B) intra-articular procedure replaces the anteromedial and posterolateral bulks (Fig. 1a and b).

The extra-articular procedures and combined intra/ extra-articular procedures were introduced by Lemaire in 1967 for chronic ACL ruptures and are still used. The 

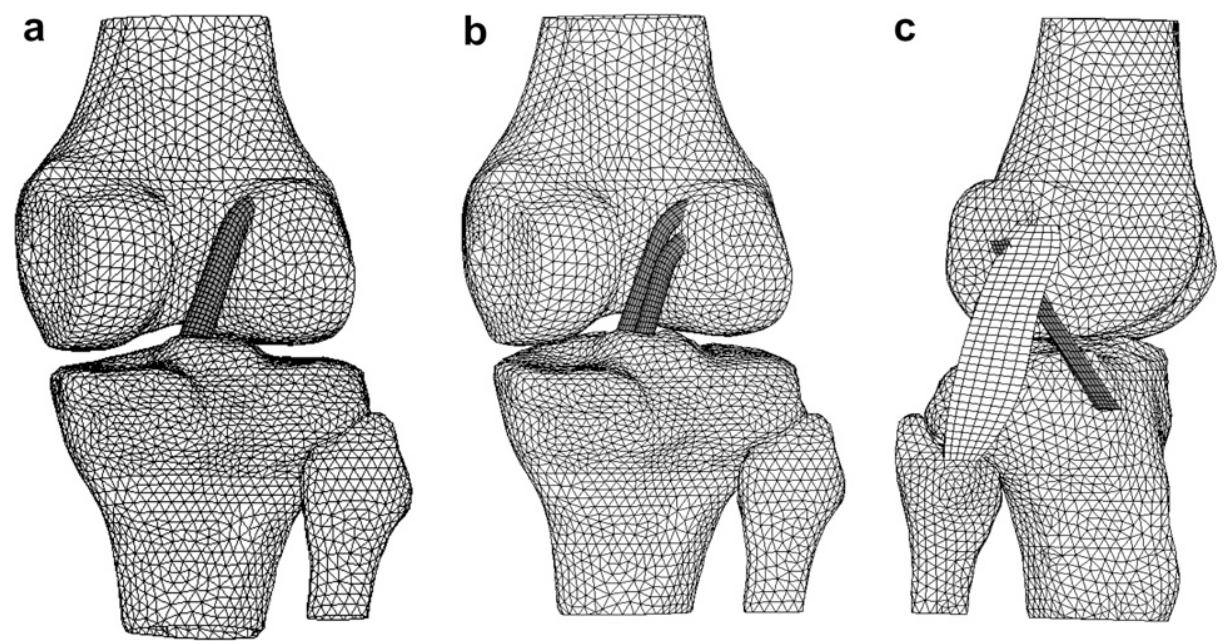

Fig. 1. (a) Single bundle intra-articular reconstruction (posterior view), (b) double bundles intra-articular reconstruction (posterior view), and (c) extraarticular reconstruction including the femur, tibia, extra-articular graft which slipped under the lateral collateral ligament and lateral collateral ligament (lateral view). For clarity, cartilages, meniscus, patellar tendons and patella are not shown in the figures.

extra-articular procedure uses a band of fascia lata distally based on Gerdy's tubercle. This band is slipped under the lateral collateral ligament and then threaded proximally through a femoral tunnel (Fig. 1c).

Clinical and experimental results found in previous studies on both types of reconstruction seem inconclusive. Biomechanical analysis of intra-articular procedures is well documented in the literature (Amis and Scammell, 1993; Yagi et al., 2002). The 2B reconstruction seems to better restore the kinematics and biomechanics of the knee when compared with the 1B reconstruction (Christel et al., 2005). But, few studies were conducted on the biomechanical analysis of the extra-articular procedure combined or not with an intra-articular one.

Extra-articular combined or not with intra-articular procedures were performed in patients with chronic ACL deficiency and in soccer players (Bak et al., 2001; Jorgensen et al., 2001). It was found that combined procedures could restore knee stability and function in the majority of these ACL deficiency knees. Other studies suggested that combined procedures provided support to the healing intraarticular graft by reducing deleterious forces and tibial displacements (McGuire and Wolchock, 2000; Noyes and Barber, 1991).

However, the clinically observed benefits of combined procedure were not confirmed in biomechanical studies (Amis and Scammell, 1993; Matsumoto and Seedhom, 1994). In these studies, the authors have examined intraarticular and combined reconstructions with cadaver knees and evaluated their effects on tibial rotation by analyzing knee movements under valgus and rotatory torques. They found that the range of tibial rotation was restored to almost its normal value when the intra-articular reconstruction was performed. Intra-articular reconstruction seemed the best way for eliminating the pivot shift (combination of valgus and internal rotation) and restoring the joint mechanics to normality. The combined reconstruc- tions did not provide additional stability. Similar clinical results were obtained where no demonstrable benefit with combined stabilization procedures for chronic ACL instability was found (Anderson et al., 2001; Strum et al., 1989).

The goal of this study is to evaluate the benefits of an extra-articular reconstruction compared to the standard intra-articular procedure. For this reason, different finite elements models were developed and used. We have considered a healthy knee, single and double bundles intra-articular reconstructions, extra-articular reconstruction alone and extra-articular combined with intra-articular reconstructions.

\section{Methods}

The 3D numerical reconstruction of the knee joint was developed in a previous study to evaluate the effects of posterior cruciate ligament (PCL) reconstruction on the biomechanics and kinematics of the knee (Ramaniraka et al., 2005). The obtained results were in agreement with clinical studies (Harner et al., 2001; Skyhar et al., 1993).

Data acquisitions were performed on the right knee of a volunteer. The knee was immobilized in full extension inside a plaster cast avoiding any movement during magnetic resonance (MR) and computed tomographyscanner (CT) images acquisition. Eight points of references were placed on the lower limb (four points on the femur and four points on the tibia) in order to match the 3D geometrical models of bone and soft structures reconstructed from CT-scanner and MR images. The markers were fixed with large scotch band in order to avoid any movements during images acquisition. Amira 3.0 (Mercury/TGS, USA) software was used for semi-automatic segmentation of MRI and CT-scanner slices. The external contour of bones and soft tissues were then accurately defined on each CT and MR slices with a digitization less than $0.8 \mathrm{~mm}$ (2 pixels). Accordingly, the accuracy of the pair point 
matching between $\mathrm{CT}$ and $\mathrm{MR}$ images registration was also $0.8 \mathrm{~mm}$.

The 3D geometry of soft tissues (ligaments, menisci, patellar tendon, and cartilage layers of tibia, femur and patella) was obtained from MR acquisitions. The CT images provided data for 3D geometry reconstruction of bone structures (tibia, femur, patella and fibula).

Bone structures were meshed with rigid surface elements due to their small strain compared to soft structures. Soft structures (cruciate ligaments, collateral ligaments, patellar tendon, and cartilage layers) were meshed with 3D hexahedral deformable elements. The numerical model used in this study contains about 36,500 elements (Fig. 1).

\subsection{Biomechanical laws}

The ligaments and the patellar tendon were modelled with non-linear hyperelastic law corresponding to the strain energy developed in Pioletti et al. (1998):

$W_{e}=\alpha \exp \left[\beta\left(I_{1}-3\right)-\frac{\alpha \beta}{2}\left(I_{2}-1\right)\right]$

where $\alpha$ and $\beta$ are material constants, and $I_{1}$ and $I_{2}$ are the strain invariants.

$I_{1}=\operatorname{tr}[C]$

$I_{2}=\frac{1}{2}\left([\operatorname{tr} C]^{2}-\operatorname{tr}[C]^{2}\right)$

$C=F^{\mathrm{T}} F$ is the (right Cauchy-Green) material metric tensor, where $F=\partial y / \partial x$ is the gradient deformation tensor.

The mean values of $\alpha$ and $\beta$ were obtained from the experimental measurements and reported in Table 1 (Pioletti and Rakotomanana, 2000).

The mechanical property of patellar tendon was used for the grafts Table 2 .

The cartilage layers of the tibia, femur and patella were considered as homogenous isotropic materials (Moglo and Shirazi-Adl, 2003). The mechanical properties of the collateral ligaments were obtained from previous works (Weiss and Gardiner, 2001).

Table 1

Mean values of the parameters $\alpha$ and $\beta$

\begin{tabular}{lll}
\hline & $\alpha[\mathrm{MPa}]$ & $\beta$ \\
\hline Anterior cruciate ligament & 0.30 & 12.20 \\
Posterior cruciate ligament & 0.18 & 17.35 \\
Patellar tendon & 0.09 & 66.96 \\
\hline
\end{tabular}

Table 2

Mechanical properties of cartilage and collateral ligaments

\begin{tabular}{lcl}
\hline & Young modulus & Poisson ratio \\
\hline Cartilage & 12 & 0.3 \\
Medial collateral ligaments & 332 & 0.4 \\
Lateral collateral ligaments & 345 & 0.4 \\
\hline
\end{tabular}

\subsection{Numerical implementation}

The numerical simulations were performed with ABAQUS/Standard 6.3 software (Hibbit, Karlsson and Sorensen Inc., Pawtucket, RI, USA). The model was used to calculate the internal rotation and the forces within the ligaments induced by an internal torque of $2 \mathrm{Nm}$. This value was chosen to compare the numerical results to the existing experimental works (Guardamagna et al., 2004).

During a normal walking, the amplitude of flexion is usually smaller than $45^{\circ}$. In the present work, we were then interested to evaluate the different mechanical situations till a maximum of $45^{\circ}$. Seven cases were simulated. We considered a knee with

(a) an intact ACL;

(b) single bundle intra-articular reconstructed ACL: the femoral and tibial attachments of the bundle graft were located within the native anteromedial bulk of the ACL insertion sites. The diameter of the graft was set to $8 \mathrm{~mm}$;

(c) double bundles intra-articular reconstructed ACL: the attachments of the bundles graft were located within the native anteromedial and posterolateral bulks of the ACL insertion sites. The graft diameters were set to $5 \mathrm{~mm}$;

(d) extra-articular procedure alone: a $5 \mathrm{~mm}$ width band distally based on Gerdy's tubercle, was slipped under the lateral collateral ligament and then threaded fixed proximally to the posterolateral part of the femur;

(e) combined extra-articular and single bundle intra-articular $A C L$ : the insertion zones of the intra-articular graft were that of single bundle intra-articular reconstruction;

(f) combined extra-articular and double bundles intraarticular ACL: the femoral and tibial insertion zones of intra-articular grafts were that of double bundles intra-articular reconstruction.

\subsection{Boundary conditions}

The tibiofemoral, patellofemoral, meniscofemoral contacts and contact between the ACL and the PCL were modeled with discontinuous unilateral large sliding laws. Moreover, bone meshes were needed to model the corresponding contact with collateral ligaments $(\mathrm{MCL} / \mathrm{femur}$, MCL/tibia, LCL/femur and LCL/tibia).

The loading condition was divided into two steps: in the first step, the femur was flexed at different angles $\left(0^{\circ}, 15^{\circ}\right.$, $30^{\circ}$ and $45^{\circ}$ ) by simulation, and in the second step, the femur was fixed and an internal torque of $2 \mathrm{Nm}$ was applied to the tibia. The tibia was fixed in the 6 degrees of freedom (three translations, three rotations) during the first step, and free in 5 degrees of freedom (three translations, internal/external rotation, varus/valgus) during the second step. All ligaments were considered stress free at $0^{\circ}$ of knee flexion. 


\section{Results}

We have calculated (a) the internal rotation angle of the tibia and (b) the forces within the ligaments (ACL, PCL, MCL and LCL) and grafts with an internal torque of $2 \mathrm{~N} \mathrm{~m}$ at $0^{\circ}, 15^{\circ}, 30^{\circ}$ and $45^{\circ}$ of knee flexion. The different positions of the bones were calculated and compared with the initial positions. The initial position of the knee was at $0^{\circ}$ of flexion.

\subsection{Internal rotation versus angles of knee flexion (Fig. 2)}

With the intact ACL, the internal rotation was maximal at $15^{\circ}$ of flexion, and, then decreased with increasing angle of knee flexion. The internal rotation angles remained between $12^{\circ}$ and $13^{\circ}$.

For both intra-articular 1B and $2 \mathrm{~B}$ reconstructions, the internal rotation was maximal at $15^{\circ}$ of flexion and, then decreased with increasing angle of knee flexion, the minimal internal rotation angles were obtained at $45^{\circ}$ of flexion.

No significant differences (lesser than $2^{\circ}$ of difference) were found on the internal rotation angles with intact, 1B and $2 \mathrm{~B}$ reconstructed $\mathrm{ACL}$ at tested angles of knee flexion.

The values of internal rotation were similar for extraarticular alone, extra-articular combined with 1B reconstruction and extra-articular combined with $2 \mathrm{~B}$ reconstruction. Internal rotation was maximal at $0^{\circ}$ and $15^{\circ}$ of knee flexion angle and then decreased with increasing flexion angle.

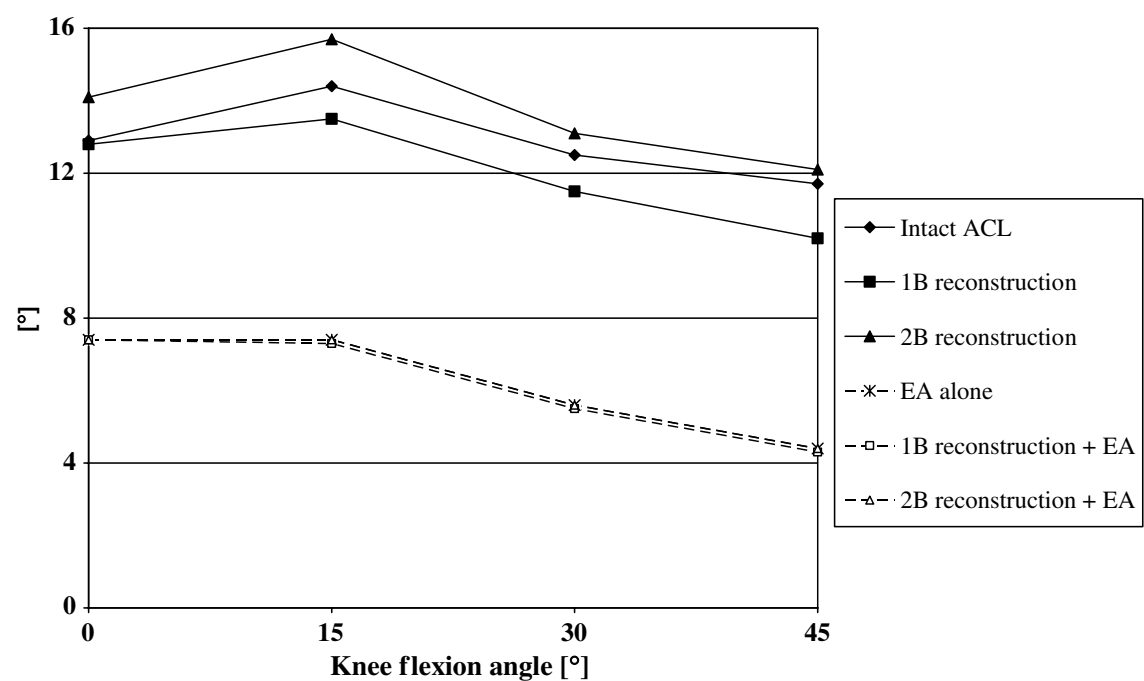

Fig. 2. Angle of internal rotation versus knee flexion angle with an intact anterior cruciate ligament, single bundle reconstruction, double bundles reconstruction, extra-articular alone, single bundle combined with extra-articular reconstruction, double bundles combined with extra-articular reconstruction.

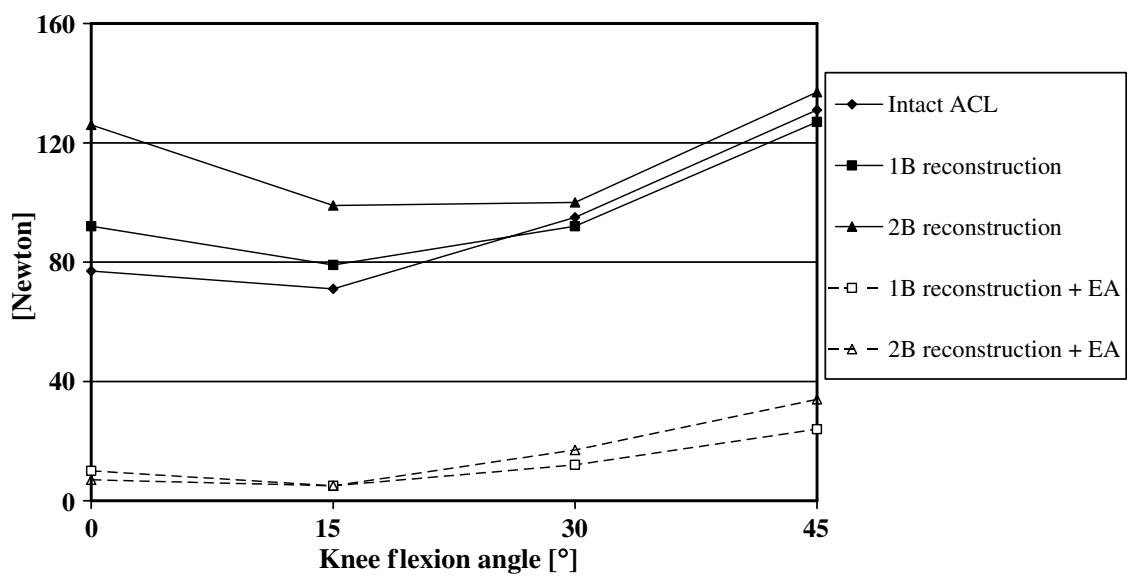

Fig. 3. Force within the anterior cruciate ligament and anterior cruciate ligament graft versus angle of knee flexion with intact anterior cruciate ligament, single bundle reconstruction alone, double bundles reconstruction alone, single bundle reconstruction combined with extra-articular procedure, and double bundles reconstruction combined with extra-articular procedure at $0^{\circ}, 15^{\circ}, 30^{\circ}, 45^{\circ}$ of knee flexion. 
3.2. Force within $A C L$ and $A C L$ grafts versus angles of knee flexion (Fig. 3)

For the intact ACL, the force magnitude in the ACL was minimal $(71 \mathrm{~N})$ at $15^{\circ}$, and maximal $(131 \mathrm{~N})$ at $45^{\circ}$ of flexion. At $0^{\circ}$ of knee flexion, this force was $77 \mathrm{~N}$.

For the 1B reconstruction, the magnitude of the force in the ACL graft was also minimal $(79 \mathrm{~N})$ at $15^{\circ}$ and maximal $(127 \mathrm{~N})$ at $45^{\circ}$ of flexion. At $0^{\circ}$ of knee flexion, the force within the graft was $92 \mathrm{~N}$.

For the $2 \mathrm{~B}$ reconstruction, the forces inside the anteromedial bundle were minimal $(37 \mathrm{~N})$ at $0^{\circ}$ of knee flexion, and the forces inside the posterolateral bundle were maximal $(89 \mathrm{~N})$ at $0^{\circ}$ of knee flexion.

For 2B reconstruction, the proportion of forces carried by the anteromedial bundle was $30 \%$ of the total force in the ACL graft at $0^{\circ}$ of knee flexion. This proportion increased with increasing knee flexion $\left(80 \%\right.$ at $15^{\circ}$, and $90 \%$ at $45^{\circ}$ of flexion).

By comparing the forces within ACL and ACL grafts, at $0^{\circ}$ of knee flexion, the force within the grafts with the $2 \mathrm{~B}$ grafts $(126 \mathrm{~N})$ was higher when compared to the intact $(77 \mathrm{~N})$ and $1 \mathrm{~B}$ graft $(92 \mathrm{~N})$.

The extra-articular procedure combined or not with intra-articular procedure decreased the force inside the ACL grafts. The extra-articular graft supported the main part of the forces.

For the extra-articular combined with $1 \mathrm{~B}$ reconstruction, the force inside the ACL graft was minimal $(5 \mathrm{~N})$ at $15^{\circ}$ and maximal $(24 \mathrm{~N})$ at $45^{\circ}$. For the extra-articular combined with $2 \mathrm{~B}$ reconstruction, these forces were $7 \mathrm{~N}$ at $0^{\circ}$ and $34 \mathrm{~N}$ at $45^{\circ}$, respectively.

\subsection{Force within the extra-articular graft (Fig. 4)}

For extra-articular procedure alone and extra-articular combined with intra-articular procedures (1B and $2 \mathrm{~B}$ ), the forces within the extra-articular graft were minimal at $0^{\circ}$ of knee flexion and maximal at $45^{\circ}$.

\subsection{Force within the collateral ligaments versus angles of knee flexion (Fig. 5)}

With the intact ACL, the MCL supported a proportion of $90 \%$ of the total force in the collateral ligaments $(\mathrm{MCL}+\mathrm{LCL})$ at different angles of flexion $(91 \%, 94 \%$, $95 \%$ and $88 \%$ at $0^{\circ}, 15^{\circ}, 30^{\circ}$ and $45^{\circ}$ of flexion, respectively).

For intra-articular (1B and 2B) reconstructions, the force in the MCL decreased with increasing angles of knee flexion. The force within the LCL was minimal $(2 \mathrm{~N})$ at $15^{\circ}$ of knee flexion.

For 2B reconstruction, the force in the MCL graft decreased with increasing angles of knee flexion, while the force within the LCL was maximal at $0^{\circ}$ and minimal at $30^{\circ}$.

For extra-articular procedure, combined or not with intra-articular reconstruction, the forces within the MCL were minimal at $30^{\circ}$ of knee flexion.

\subsection{Force within the posterior cruciate ligament versus angles of knee flexion (Fig. 6)}

The force in the PCL is mainly due, on one hand, to the flexion during the first step of the applied external conditions and, in the other hand, to the impingement between the ACL and the PCL during the internal torque. In the four cases (healthy knee, resected ACL, 1B and 2B reconstructions), the force within the PCL was minimal at $15^{\circ}$ of flexion ( $28 \mathrm{~N}$ with an intact ACL, $25 \mathrm{~N}$ with $1 \mathrm{~B}$ reconstruction, and $22 \mathrm{~N}$ with $2 \mathrm{~B}$ reconstruction). This force was maximal at $45^{\circ}$ of flexion $(121 \mathrm{~N}$ with an intact ACL, $16 \mathrm{~N}$ with a resected ACL, $126 \mathrm{~N}$ with $1 \mathrm{~B}$ reconstruction, and $107 \mathrm{~N}$ with $2 \mathrm{~B}$ reconstruction).

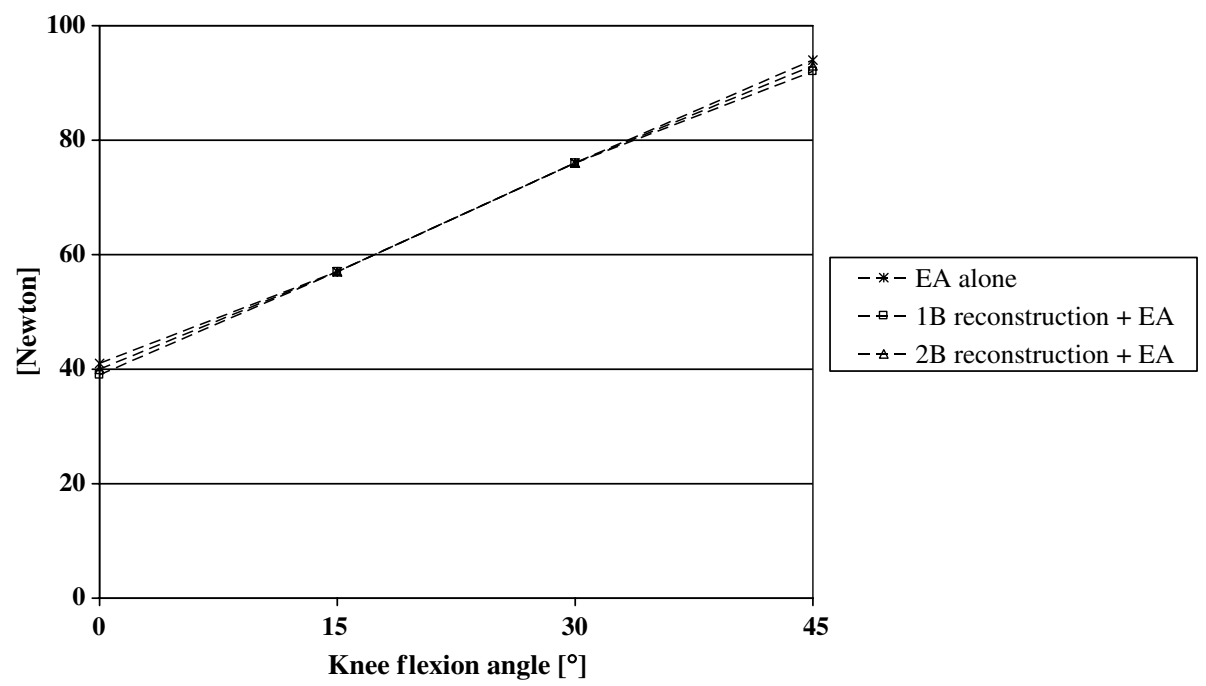

Fig. 4. Force inside the extra-articular grafts at different angles of knee flexion with (a) extra-articular reconstruction alone, (b) single bundle combined with extra-articular reconstruction, and (c) double bundles combined with extra-articular reconstruction. 


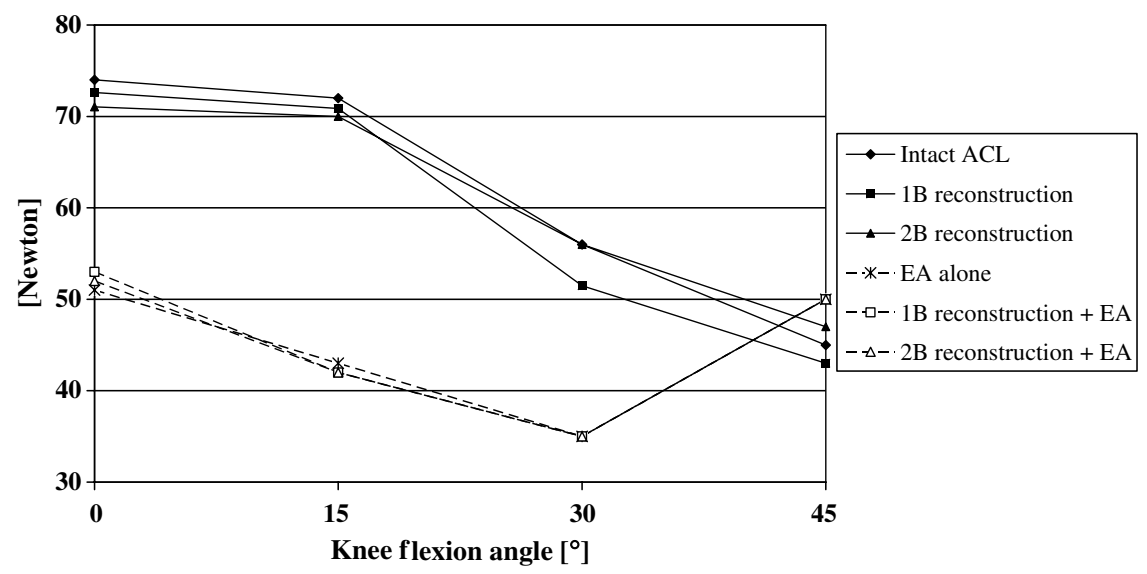

Fig. 5. Force inside medial collateral ligament with (a) intact anterior cruciate ligament, (b) single bundle reconstruction, (c) double bundles reconstruction, (d) extra-articular reconstruction alone, (e) extra-articular combined with single bundle reconstruction, and (f) extra-articular combined double bundles reconstruction at $15^{\circ}$ of knee flexion.

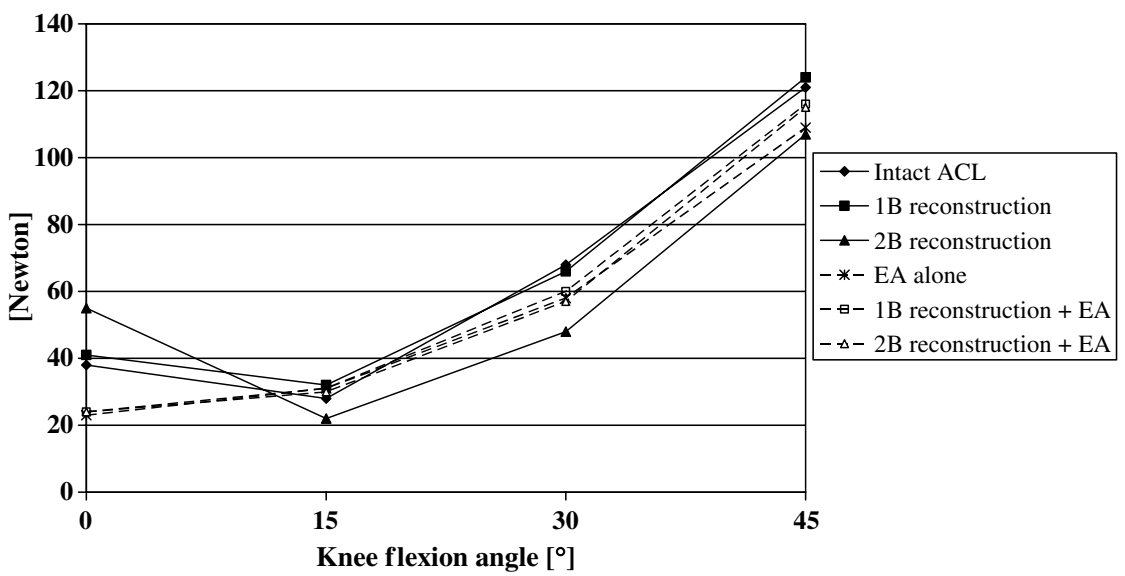

Fig. 6. Force inside posterior cruciate ligament with (a) intact anterior cruciate ligament, (b) single bundle reconstruction, (c) double bundles reconstruction, (d) extra-articular reconstruction alone, (e) extra-articular combined with single bundle reconstruction, and (f) extra-articular combined double bundles reconstruction at $15^{\circ}$ of knee flexion.

With extra-articular procedure, combined or not with intra-articular reconstruction, the forces within the PCL were minimal at $0^{\circ}$ of knee flexion $(23 \mathrm{~N}, 24 \mathrm{~N}$ and $24 \mathrm{~N}$ with extra-articular alone, extra-articular combined with $1 \mathrm{~B}$ and extra-articular combined with $2 \mathrm{~B}$ reconstruction). These forces were maximal at $45^{\circ}$ of knee flexion $(109 \mathrm{~N}$, $116 \mathrm{~N}$ and $115 \mathrm{~N}$ with extra-articular alone, extra-articular combined with $1 \mathrm{~B}$ and extra-articular combined with $2 \mathrm{~B}$ reconstruction).

\section{Discussion}

The aims of the present study were to evaluate the effects of the types of ACL reconstruction (intra, extra-articular and combined procedures) on the biomechanics and kinematics of the knee. The internal rotations, the stresses inside the ligaments and grafts were calculated when an internal torque was applied. This is the first study that numerically compared the effectiveness of intra-articular and extra-articular ACL reconstructions on the kinematics and biomechanics of the knee during an internal rotation test.

We found that the extra-articular procedure combined or not with the intra-articular procedure decreased about $50 \%$, the internal rotation of the tibia at tested angles of knee flexion. Our results showed that an intra-articular reconstruction restore better the internal rotation than the extra-articular procedure. Moreover, our results are in agreement with experimental measurements (Guardamagna et al., 2004). No significant differences were found in internal rotation between the healthy knee and intraarticular (1B and 2B) reconstructed ACL at tested angles of knee flexion. The intra-articular procedure seems to be sufficient to restore the kinematics of the knee during internal rotation test. However, it was suggested that a severe rotatory stability could be restored with the extra-articular procedure (McGuire and Wolchock, 2000). In their works, they performed extra-articular procedure with patients 
whose lateral structures are compromised with ACL disruption. In our study, we have considered that lateral structures were not compromised.

Concerning the extra-articular procedure, it has been suggested that the extra-articular procedure could produce a satisfactory result 3-4 years after operation in the majority of patients (Thomas et al., 1998). It was found that the extra-articular procedure results in a lower deficit for the peak quadriceps and hamstring torques and the range of motion.

Concerning the forces in the ACL and ACL grafts with single bundle reconstruction ACL, we found that the extraarticular graft supported the greatest proportion of forces to restrain the internal rotation. Our results are in agreement with the previous works (Noyes and Barber, 1991), the extra-articular structure provided support to the healing intra-articular graft by reducing deleterious forces. However, the forces within the ACL grafts with intra-articular alone were similar to the forces in the ACL of a healthy knee.

Previous experimental study have shown that the force in the posterolateral bundle decrease from $0^{\circ}$ to $45^{\circ}$ of flexion (Guardamagna et al., 2004). Moreover, as previously observed, the $2 \mathrm{~B}$ reconstruction should allow for a better reconstruction than the $1 \mathrm{~B}$ procedure, the posterolateral bundle is responsible for controlling internal rotation of the tibia (Christel et al., 2005). Our results are in agreement to these experimental works (Gabriel et al., 2004; Guardamagna et al., 2004), the posterolateral bundle of $2 \mathrm{~B}$ reconstruction is the primary restraint to internal rotation near $0^{\circ}$ of knee flexion. Otherwise, the anteromedial bundle carries the greatest proportion of force in the ACL graft.

With an extra-articular procedure, in our study, the forces in the MCL were altered. These forces decreased from $0^{\circ}$ to $30^{\circ}$ of knee flexion and then increased. By comparing with the intra-articular procedure, the forces within the MCL decreased from $0^{\circ}$ to $30^{\circ}$. The extra-articular graft was the principal structure responsible to restrain the internal rotation.

By comparing the forces within the collateral ligaments, with intact and intra-articular reconstructed ACL procedure, our results are similar to previous findings (Amis et al., 2005; Fu et al., 1993) where the MCL were the major restraints to internal tibial rotation. In our study, we found that a resection of the ACL alters the force distribution within collateral ligaments when compared with an intact knee. The LCL seems to have a role in limiting internal tibial rotation only near the $0^{\circ}$ of knee flexion. This force within the LCL decreases as the knee is flexed, as seen in previous study (Meister et al., 2000).

The extra-articular procedure has not altered appreciably the forces in the PCL when compared with the intraarticular procedure. These results could show that the extra-articular procedure did not affect the role of the PCL in the internal rotation test. As seen in a previous study (Larson, 2001), the PCL has not a significant role in internal rotation.
The present study has a number of limitations. We used only loading corresponding to a combined rotatory load (flexion and internal tibial rotation) which did not constitute the standard test for ACL reconstruction. Other types of loading, such as combination of internal/external rotation with anterior tibial translation and varus/valgus rotation, could give supplementary information on the effects of the ACL reconstruction. During ACL reconstruction, a pre-tension is applied to the graft at near $45^{\circ}$ of knee flexion. Different values of pre-tension are proposed in the literature but no consensus was found on the optimal values of pre-tension to be applied.

In the present study, we have tested a stress-free initial condition to be in conformity with the experimental study (Guardamagna et al., 2004), but initial pre-stress could certainly modify the stress inside the ligaments and grafts. The present model could simulate the ACL reconstruction with an initial pre-tension. Finally, the effects of bone morphology were not evaluated in the present study. However, the morphology of the considered knee corresponded to the standard morphology of a Caucasian male knee.

\section{Conclusion}

In this numerical study, we showed that the extra-articular procedure alters the kinematics and the biomechanics of the knee. The intra-articular procedure is the best way to restore the biomechanics and kinematics of the knee. With this procedure, the internal rotation of the knee and stresses inside the graft, obtained with intra-articular reconstructions, are closed to the situation with the intact ACL.

\section{Acknowledgement}

The authors acknowledge the financial support provided by the Swiss National Science Foundation Grant No. 3200B0-105976/1.

\section{References}

Amis, A.A., Scammell, B.E., 1993. Biomechanics of intra-articular and extra-articular reconstruction of the anterior cruciate ligament. J. Bone Joint Surg. 75B, 812-818.

Amis, A.A., Bull, A.M.J., Lie, D.T.T., 2005. Biomechanics of rotational instability and anatomic ACL reconstruction. Oper. Techn. Orthop., 29-35.

Anderson, A.F., Snyder, R.B., Lipscomb, A.B., 2001. Anterior cruciate ligament reconstruction. A prospective randomized study of three surgical methods. Am. J. Sports Med. 29 (3), 272-279.

Bak, K., Jorgensen, U., Ekstrand, J., Scavenius, M., 2001. Reconstruction of anterior cruciate ligament deficient knees in soccer players with an iliotibial band autograft. A prospective study of 132 reconstructed knees followed for 4 (2-7) years. Scand. J. Med. Sci. Sports 11, 16-22.

Christel, P., Franceschi, J.P., Sbihi, A., Colombet, P., Djian, P., Bellier, G., 2005. Anatomic acl reconstruction: the French experience. Oper. Techn. Orthop. 15, 103-110.

Fu, F.H., Harner, C.D., Johnson, D.L., Miller, M.D., Woo, S.L.Y., 1993 Biomechanics of knee ligaments. Basic concepts and clinical application. J. Bone Joint Surg. 75A, 1716-1727. 
Gabriel, M.T., Wong, E.K., Woo, S.L.Y., Yagi, M., Debski, R.E., 2004. Distribution of in situ forces in the anterior cruciate ligament in response to rotatory loads. J. Orthop. Res. 22, 85-89.

Guardamagna, L., Seedhom, B.B., Ostell, A.E., 2004. Double-band reconstruction of the ACL using a synthetic implant: a cadaveric study of knee laxity. J. Orthop. Sci. 9, 372-379.

Harner, C.D., Vogrin, T.M., Woo, S.L-Y., 2001. Anatomy and biomechanics of the posterior cruciate ligament. In: Gregory, C. (Ed.), Posterior Cruciate Injuries: A Practical Guide to Management. Springer, Fanelli.

Jorgensen, U., Bak, K., Ekstrand, J., Scavenius, M., 2001. Reconstruction of the anterior cruciate ligament with the ilioband autograft in patient with chronic knee instability. Knee Surg. Sports Traumatol. Arthrosc. 9, 137-145.

Larson, R.V., 2001. Clinical evaluation of posterior cruciate ligament and posterolateral corner insufficiency. Oper. Techn. Sports Med. 9, 47-52.

Matsumoto, H., Seedhom, B.B., 1994. Treatment of the pivot-shift intraarticular versus extra-articular or combined reconstruction procedures A biomechanical study. Clin. Orthop. Relat. Res. 299, 298-304.

McGuire, D.A., Wolchock, J.C., 2000. Extra-articular lateral reconstruction technique. Arthroscopy 5, 553-557.

Meister, B.E., Michael, S.P., Moyer, R.A., Kelly, J.D., Schneck, C.D., 2000. Anatomy and kinematics of the lateral collateral ligament of the knee. Am. J. Sports Med. 28, 869-878.

Moglo, K.E., Shirazi-Adl, A., 2003. On the coupling between anterior and posterior cruciate ligaments, and knee joint response under anterior femoral drawer in flexion: a finite element study. Clin. Biomech. 18, $751-759$.
Noyes, F.R., Barber, S.D., 1991. The effect of an extra-articular procedure on allograft reconstructions for chronic ruptures of the anterior cruciate ligament. J. Bone Joint Surg. 73-A, 882-892.

Pioletti, D.P., Rakotomanana, L.R., 2000. Non-linear viscoelastic law for soft biological tissues. Eur. J. Mech. 19, 749-759.

Pioletti, D.P., Rakotomanana, L.R., Benvenuti, J.F., Leyvraz, P.-F., 1998. Viscoelastic constitutive law in large deformations: application to human knee ligaments and tendons. J. Biomech. 31, 753-757.

Ramaniraka, N.A., Terrier, A., Theumann, N., Siegrist, O., 2005. Effects of the posterior cruciate ligament reconstruction on the biomechanics of the knee joint: a finite element analysis. Clin. Biomech. 20, 434-442.

Skyhar, M.J., Warren, R.F., Ortiz, G.J., Schwartz, E., Otis, J.C., 1993. The effects of sectioning of the PCL and the posterolateral complex on the articular contact pressures within the knee. J. Bone Joint Surg. 75A (5), 694-699.

Strum, G.M., Fox, J.M., Ferkel, R.D., Dorey, F.H., Del Pizzo, W., Friedman, M.J., Snyder, S.J., Markolf, K., 1989. Intra-articular versus intra-articular and extra-articular reconstruction for chronic anterior cruciate ligament instability. Clin. Orthop. Relat. Res. 245, 188-198.

Thomas, O.L., Oni, O.A., Howard, L., 1998. Clinical evaluation of the Lemaire anterior concrete lateral substitution procedure. A quality audit of the Leicester modification. Injury 29, 417-419.

Weiss, J.A., Gardiner, J.C., 2001. Computational modeling of ligaments mechanics. Crit. Rev. Biomed. Eng. 29, 1-70.

Yagi, M., Wong, E.K., Kanamori, A., Debski, R.E., Fu, F.H., Woo, S.L.Y., 2002. Biomechanical analysis of an anatomic anterior cruciate ligament reconstruction. Am. J. Sports Med. 230, 660-666. 